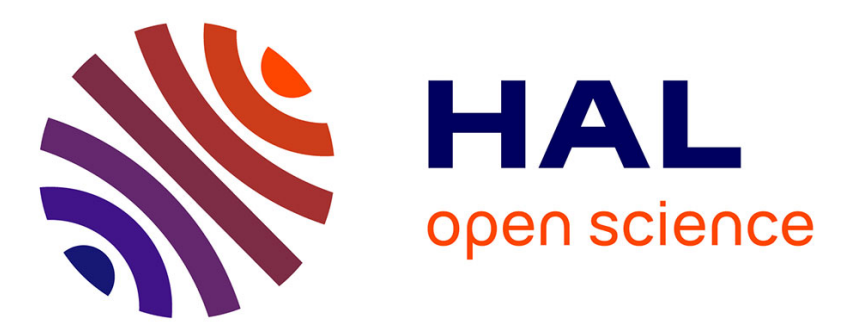

\title{
Cristallisation à basse température du polybutène-1 : étude par effet Raman
}

\author{
M. Abenoza, A. Armengaud, J. Rault
}

\section{To cite this version:}

M. Abenoza, A. Armengaud, J. Rault. Cristallisation à basse température du polybutène1: étude par effet Raman. Journal de Physique Lettres, 1979, 40 (17), pp.445-449. 10.1051/jphyslet:019790040017044500 . jpa-00231662

\section{HAL Id: jpa-00231662 https://hal.science/jpa-00231662}

Submitted on 1 Jan 1979

HAL is a multi-disciplinary open access archive for the deposit and dissemination of scientific research documents, whether they are published or not. The documents may come from teaching and research institutions in France or abroad, or from public or private research centers.
L'archive ouverte pluridisciplinaire HAL, est destinée au dépôt et à la diffusion de documents scientifiques de niveau recherche, publiés ou non, émanant des établissements d'enseignement et de recherche français ou étrangers, des laboratoires publics ou privés. 


\title{
Cristallisation à basse température du polybutène-1 : étude par effet Raman
}

\author{
M. Abenoza, A. Armengaud \\ Laboratoire de Spectroscopie Raman I, Université des Sciences et Techniques du Languedoc, 34060 Montpellier, France \\ et J. Rault
}

Laboratoire de Physique des Solides, Université Paris-Sud, 91405 Orsay, France

(Reçu le 12 avril 1979, révisé le 26 juin 1979, accepté le 5 juillet 1979)

\begin{abstract}
Résumé. - L'étude de la variation avec la température de la polarisation et des hauteurs relatives des raies du spectre Raman d'un verre de polybutène-1 obtenu par trempe dans l'azote liquide du polymère fondu, nous permet de mettre en évidence, au-dessous de la température de transition vitreuse $T_{\mathrm{G}}$, la cristallisation du matériau sous la forme cristalline instable II à $193 \mathrm{~K}$ et la transformation de cette forme vers la forme stable I à $233 \mathrm{~K}$.
\end{abstract}

\begin{abstract}
The polarization and the relative heights of the Raman bands for a melt quenched polybutene-1 glass were measured as a function of the temperature from 93 to $233 \dot{K}$. This study shows that there is a crystallization in the sample with a transition to the II crystalline modification at $193 \mathrm{~K}$ and the transformation form II $\rightarrow$ form I at $233 \mathrm{~K}$.
\end{abstract}

1. Introduction. - Le polybutène-1 (PB-1) isotactique se présente sous 3 formes cristallines polymorphes : la forme I rhomboédrique et stable [1, 4], la forme II tétragonale et instable [3, 4], et la forme III orthorhombique et stable qui ne peut être obtenue qu'à partir de solutions $[4,5]$; dans ces trois formes la molécule du polymère a respectivement la conformation des hélices $3_{1}[3,4], 11_{3}[3,4]$ et $10_{3}[6]$.

La cinétique de cristallisation du PB-1 à partir du polymère surfondu et la transformation forme II $\rightarrow$ forme I ont été étudiées, principalement par analyse thermique différentielle $[7,8]$ par dilatométrie $[7,9,10]$ ou par spectroscopie infrarouge $[7,8$, 11]. Il n'existe par contre, à notre connaissance aucune étude sur la cristallisation du PB-1 à très basse température.

Le spectre de diffusion Raman d'une substance rend compte de tous les isomères de rotation présents dans l'échantillon et de leur environnement : l'effet Raman peut donc permettre de déceler d'une part le début d'une cristallisation de l'échantillon [12] et d'autre part les modifications de formes cristallines. Dans cette étude, nous avons obtenu du PB-1 amorphe à la température de l'azote liquide et nous avons mis en évidence, compte tenu de nos conditions expérimentales (temps d'étude limité à 24 ou $48 \mathrm{~h}$ pour une température donnée selon le domaine de température, taux minimum de transformation déce- lable de 5 à $10 \%$ ), une transition à $193 \mathrm{~K}$, température correspondant à la limite inférieure du domaine de cristallisation primaire et une transformation phase II $\rightarrow$ phase I, lente et graduelle, qui débute vers $223 \mathrm{~K}$.

2. Conditions expérimentales. - Les spectres Raman ont été enregistrés sur un spectromètre de haute résolution, à double monochromateur, mis au point au Laboratoire [13] ; la technique utilisée est la diffusion à $90^{\circ}$ en incidence normale, l'excitatrice étant la radiation $5145 \AA$ d'un laser Spectra Physics à argon ionisé (puissance utilisée : 700 à $900 \mathrm{~mW}$ ), polarisée perpendiculairement au plan de diffusion; le faisceau diffusé est polarisé soit perpendiculairement (cas $I_{\mathrm{Vv}}$ ) soit parallèlement (cas $I_{\mathrm{VH}}$ ) au plan de diffusion à l'aide d'une lame demi-onde centrée sur $5380 \AA$.

Le polybutène-1 utilisé est un produit $\mathrm{CdF}$ Chimie se présentant sous forme de pastilles translucides de $5 \mathrm{~mm}$ de diamètre et de 3 à $4 \mathrm{~mm}$ d'épaisseur. Ses caractéristiques sont :

$$
M_{\mathrm{w}}=394000, M_{\mathrm{N}}=80700 \text {; isotacticité : } 99 \% \text {. }
$$

Les pastilles de PB-1, initialement portées à une température de $433 \mathrm{~K}$ pendant $15 \mathrm{~min}$., sont instantanément trempées dans l'azote liquide puis placées, 
à $93 \mathrm{~K}$, dans un cryostat Coderg, de type « Cryocir ", dont la température déterminée par une sonde à résistance est stabilisée à $2 \mathrm{~K}$ près. Les spectres Raman ont été enregistrés tous les $20 \mathrm{~K}$ de 93 à $193 \mathrm{~K}$, puis tous les $10 \mathrm{~K}$, de 193 à $233 \mathrm{~K}$. La figure 1 donne les spectres de ces échantillons aux températures de $93,193,223$ et $233 \mathrm{~K}$ pour des polarisations du faisceau diffusé respectivement perpendiculaire et parallèle au plan de diffusion.

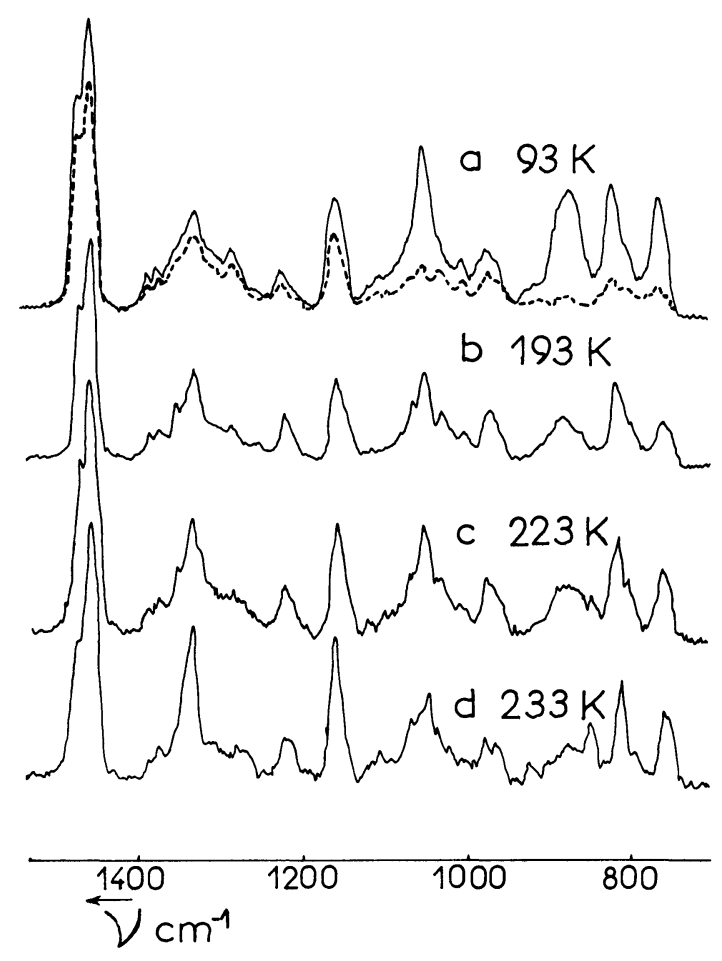

Fig. 1. - Spectres Raman à différentes températures d'un échantillon de polybutène- 1 obtenu par trempe dans l'azote liquide du polymère fondu. — polarisation du faisceau diffusé perpendiculaire au plan de diffusion ; - . - polarisation du faisceau diffusé parallèle au plan de diffusion. N.B. : pour les spectres b, c, d, les courbes en trait plein et en pointillés étant pratiquement confondues, seule la courbe en trait plein a été présentée.

[Raman spectra of melt quenched polybutene-1 sample at various temperatures : — scattering light polarization normal to the scattering plane ; - - - scattering light polarization parallel to the scattering plane.]

Les spectres relatifs aux différentes phases connues du polybutène (Fig. 2) ont été pris dans les conditions ci-après :

— pastille de PB-1 à température ordinaire pour la forme cristalline $I$;

- pastille de PB-1, placée dans une cuve parallélipipédique scellée sous vide pour éviter toute oxydation du polymère, portée d'abord à $433 \mathrm{~K}$ pendant plusieurs minutes, puis à $398 \mathrm{~K}$ et sous le faisceau du laser pendant $3 \mathrm{~h}$ afin d'éliminer les bandes de fluorescence, pour la phase amorphe ;

- échantillon précédent (amorphe fondu) porté à $373 \mathrm{~K}$ pendant $24 \mathrm{~h}$, pour obtenir la forme cristalline II.

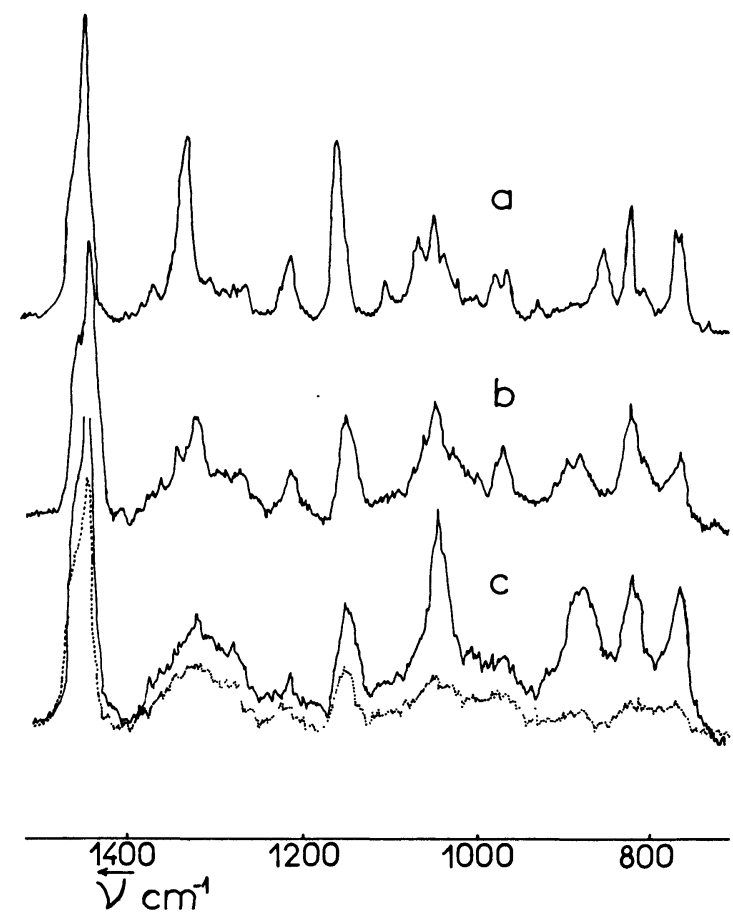

Fig. 2. - Spectres Raman du polybutène-1:a) forme cristalline I ; b) forme cristalline II ; c) amorphe liquide :- polarisation du faisceau diffusé perpendiculaire au plan de diffusion $; \ldots \ldots$ polarisation du faisceau diffusé parallèle au plan de diffusion. N.B. : pour les formes cristallines I et II, les courbes en trait plein et en pointillés étant pratiquement confondues, seule la courbe en trait plein a été présentée (voir les valeurs de $\rho$ dans le tableau I).

[Raman spectra of polybutene-1 : a) crystalline modification I ; b) crystalline modification II ; c) melt : - scattering light polarization normal to the scattering plane ; ..... scattering light polarization parallel to the scattering plane.]

Les conditions expérimentales et la transparence de l'échantillon n'étant pas les mêmes pour les 3 phases, il n'est pas possible de comparer entre elles les intensités des raies des spectres obtenus pour les différentes phases.

Nous avons alors caractérisé chaque phase par les hauteurs relatives $h$ des raies calculées par rapport à la hauteur de la raie de fréquence $1445 \mathrm{~cm}^{-1}$ prise comme unité dans le cas de polarisation $I_{\mathrm{Vv}}$ et par le rapport $\rho$ des hauteurs des raies homologues des spectres correspondant au cas de polarisation $I_{\mathrm{VH}}$ et $I_{\mathrm{vv}}$.

3. Discussion des résultats. - 3.1 CARACTÉRISATION DES FORMES I ET II ET DE LA PHASE AMORPHE PAR SPECTROSCOPIE RAMAN. - La caractérisation des formes cristallines I et II du PB-1 n'a été faite, en spectroscopie Raman, qu'à partir des fréquences des raies [6]. Il nous paraît cependant nécessaire, surtout si on fait intervenir le spectre du matériau fondu dont les fréquences sont très voisines de celles de la forme cristalline II, de préciser cette étude en déterminant les hauteurs relatives et les rapports $\rho$ 
Tableau I. - Fréquences $v\left(\mathrm{~cm}^{-1}\right)$, hauteurs relatives het rapports $\rho=I_{\mathrm{VH}} / I_{\mathrm{Vv}}$ des raies Raman caractéristiques des formes cristallines I et II, de l'amorphe fondu et de l'échantillon trempé à $93 \mathrm{~K}$ de polybutène-1 :

$\mathrm{f}$ : faible ; t.f. : très faible ; abs. : absent ; ép. : épaulement.

[Frequencies $v\left(\mathrm{~cm}^{-1}\right)$, relative heights $h$ and ratios $\rho=I_{\mathrm{VH}} / I_{\mathrm{Vv}}$ of the characteristic Raman bands for the I and II crystalline modifications, and for melt and quenched samples of polybutene-1 :

f. : weak ; t.f. : very weak ; abs. : absent ; ep. : shoulder.]

Forme I

$(293 \mathrm{~K})$

$v \mathrm{~cm}^{-1}$
$1 \overline{-}$
1444
1326
1155
1096
1040
abs.
849
818
767
760

Forme II

(373 K)

$\begin{array}{ccc}v \mathrm{~cm}^{-1} & h & \rho \\ -\overline{1} & - & - \\ 1440 & 1 & 0,95 \\ 1318 & 0,36 & 1,07 \\ 1143 & 0,37 & 0,99 \\ \text { abs. } & - & - \\ 1042 & 0,40 & 0,94 \\ 870-881 & 0,23 & 0,85 \\ \text { abs. } & - & - \\ 817 & 0,40 & 0,86 \\ 767 & 0,26 & 0,74 \\ 758 & & \end{array}$

pour les raies des spectres de chacune des formes considérées. L'examen du tableau I montre que l'on peut différencier sans aucune ambiguité les spectres de la phase amorphe d'une part et ceux des formes cristallines d'autre part, donc déceler une cristallisation éventuelle, par la mesure du rapport $\rho$, particulièrement pour les raies dont la fréquence est comprise entre 750 et $1050 \mathrm{~cm}^{-1}$. Aux erreurs d'expérience près, la valeur $\rho \simeq 1$ peut être prise comme critère de cristallinité.

En outre, la transformation forme II $\rightarrow$ forme I pourra être mise en évidence, soit par l'étude de la variation des fréquences et des hauteurs relatives des raies situées à 1143 et $1318 \mathrm{~cm}^{-1}$ dans la forme II, soit par l'apparition des raies à 849 et $1096 \mathrm{~cm}^{-1}$ et la disparition de la raie large située vers $880 \mathrm{~cm}^{-1}$.

3.2 ETUDE DU MATÉRIAU OBTENU PAR TREMPE DU PB FONDU. - Les spectres du matériau obtenu par trempe à $93 \mathrm{~K}$ du PB-1 fondu (Fig. 1a) sont pratiquement identiques à ceux de l'amorphe liquide (Fig. $2 c$ ), la meilleure définition de certaines raies dans les premiers provenant de l'effet de température. Le matériau obtenu est donc un verre de PB-1.

Le tableau I montre que les rapports $\rho$ relatifs aux raies du spectre du matériau trempé sont voisins mais légèrement supérieurs à ceux des raies du spectre de l'amorphe fondu ; ces différences ne sont cependant pas significatives car $\rho$ étant déduit d'un rapport de hauteurs de raies, les différences observées peuvent résulter d'une variation de largeur de raies due à un effet de température, les intensités intégrées restant égales dans les deux spectres.

Une série d'expériences a alors été réalisée en portant une pastille de $\mathrm{PB}-1$ à $433 \mathrm{~K}$ comme précédemment, mais en laissant l'échantillon à la sortie du four à température ambiante pendant un temps
Amorphe fondu (398 K)

$\begin{array}{ccc}v \mathrm{~cm}^{-1} & h & \rho \\ - & - & - \\ 1441 & 1 & 0,74 \\ 1316 & 0,32 & 0,68 \\ 1145 & 0,35 & 0,6 \\ \text { abs. } & - & - \\ 1039 & 0,63 & 0,28 \\ 871-881 & 0,43 & \text { t.f. } \\ \text { abs. } & - & - \\ 816 & 0,46 & 0,22 \\ \text { abs. } & - & - \\ 760 & 0,46 & 0,22\end{array}$

Echantillon trempé (93 K)

$\begin{array}{ccc}v \mathrm{~cm}^{-1} & h & \rho \\ - & - & - \\ 1440 & 1 & 0,77 \\ 1316 & 0,35 & 0,78 \\ 1147 & 0,40 & 0,66 \\ \text { abs. } & - & - \\ 1041 & 0,56 & 0,28 \\ 870-880 & 0,42 & \text { t.f. } \\ \text { abs. } & - & - \\ 820 & 0,43 & 0,33 \\ \text { abs. } & - & - \\ 760 & 0,41 & 0,24\end{array}$

variant de 10 à $180 \mathrm{~s}$, avant la trempe dans l'azote liquide; les spectres Raman pris immédiatement après la trempe, à $93 \mathrm{~K}$, évoluent progressivement vers celui de la forme II : la phase amorphe est encore prédominante pour les échantillons dont l'attente à la température ambiante n'a pas dépassé un temps de l'ordre de $60 \mathrm{~s}$; par contre, les spectres des échantillons laissés $3 \mathrm{~min}$ à l'air libre sont identiques à ceux de la forme cristalline II. En aucun cas les raies caractéristiques de la forme I n'apparaissent dans les spectres. Ces résultats sont en accord avec les études antérieures qui font état d'une cristallisation très rapide du PB-1 au-dessous de $333 \mathrm{~K}$ environ $[9,10]$ et d'une cinétique de transformation II $\rightarrow$ I très lente à la température ordinaire ([11], par exemple).

3.3 EVolution du MATÉRIAU TREMPÉ DE 93 A $233 \mathrm{~K}$. - De 93 à $193 \mathrm{~K}$, les spectres ont été enregistrés $2 \mathrm{~h}, 4 \mathrm{~h}, 8 \mathrm{~h}$ et $24 \mathrm{~h}$ après l'obtention de la température considérée. Au-dessus de $193 \mathrm{~K}$, en raison d'une cinétique de transformation plus lente, l'échantillon a été maintenu pendant $48 \mathrm{~h}$ à chaque température, un spectre supplémentaire étant pris au bout de ce temps-là.

L'étude de l'ensemble des spectres montre que : - de 93 à $173 \mathrm{~K}$ les rapports $\rho$ (Fig. 3) et les hauteurs relatives (Fig. 4) des raies ne subissent aucune modification ; ces constatations sont également valables pour les échantillons dans lesquels coexistaient à $93 \mathrm{~K}$ la phase amorphe et la forme cristalline II. Aucune cristallisation n'est donc décelable par notre technique, en $24 \mathrm{~h}$, au-dessous de $173 \mathrm{~K}$;

- par contre, à $193 \mathrm{~K}$, et dès le premier spectre terminé $2 \mathrm{~h}$ après l'obtention de cette température, les valeurs des rapports sont complètement modifiés : il y a cristallisation à l'intérieur de l'échantillon. 


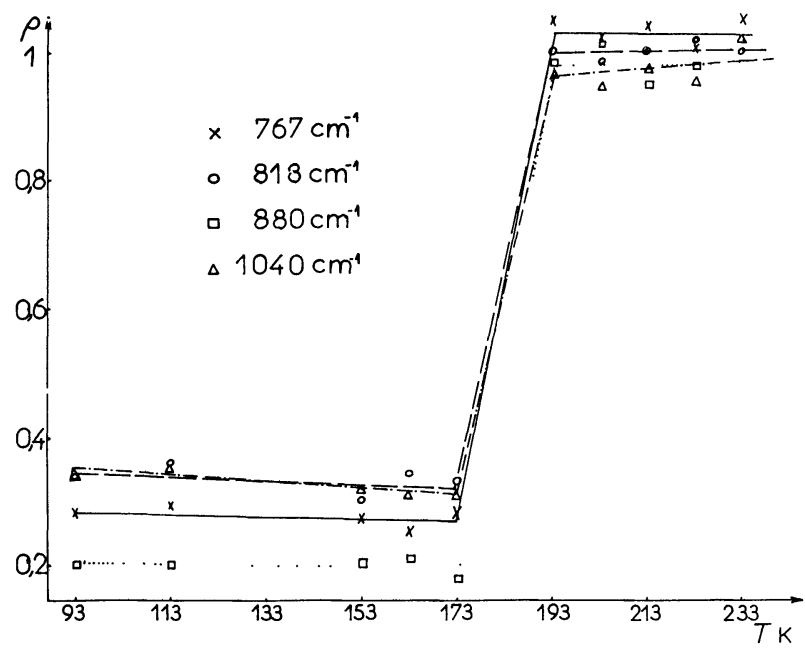

Fig. 3. - Variation du facteur $\rho=I_{\mathrm{VH}} / I_{\mathrm{vv}}$ des raies Raman de l'échantillon de PB-1 étudié en fonction de la température : raies à $767 \mathrm{~cm}^{-1}(\longrightarrow), 818 \mathrm{~cm}^{-1}(-\longrightarrow) 880 \mathrm{~cm}^{-1}(\ldots)$ et $1040 \mathrm{~cm}^{-1}$ (一. -. - ).

[Ratio $\rho=I_{\mathrm{VH}} / I_{\mathrm{Vv}}$ of polybutene-1 sample Raman bands versus temperature : $767(-), 818(-\longrightarrow), 880(\ldots)$ and $1040(-.-$. $\mathrm{cm}^{-1}$ bands.]

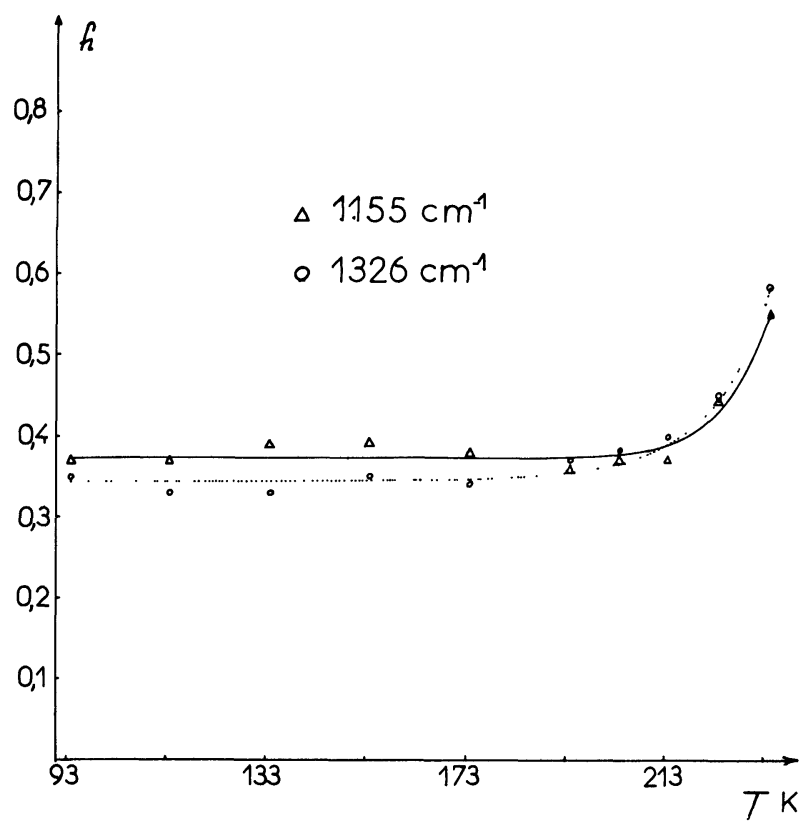

Fig. 4. - Variation des hauteurs relatives des raies situées à 1155 ( $\left(\right.$ ) et $1326 \mathrm{~cm}^{-1}(\ldots)$ en fonction de la température, pour le polybutène-1 étudié.

[Relative heights of polybutene-1 sample Raman bands at $1155 \mathrm{~cm}^{-1}(\longrightarrow)$ and $1326 \mathrm{~cm}^{-1}(\ldots)$ versus temperature.]

L'allure du spectre et la valeur des hauteurs relatives des raies montrent que c'est la forme II qui apparaît ; la transformation paraît complète après $2 \mathrm{~h}$ à cette température car les valeurs de $\rho$ atteignent les valeurs caractéristiques de la forme II obtenue dans les conditions habituelles (tableau I) ;

— de 193 à $223 \mathrm{~K}$ les spectres n'évoluent pas.
Dans ces conditions, la forme II est la seule phase cristalline décelable dans l'échantillon;

- entre 223 et $233 \mathrm{~K}$, une nouvelle modification se produit : les hauteurs relatives des raies varient progressivement avec le temps, la raie située à $880 \mathrm{~cm}^{-1}$ diminue d'intensité tandis qu'apparaissent les raies de fréquences 849 et $1096 \mathrm{~cm}^{-1}$ : la forme II se transforme lentement en forme I, la transformation à peine visible à $223 \mathrm{~K}$ est accélérée par le passage à $233 \mathrm{~K}$ et est pratiquement totale après $48 \mathrm{~h}$ à cette température.

4. Conclusion. - L'étude de la polarisation et des hauteurs relatives des raies Raman d'un échantillon de PB-1 initialement à l'état vitreux à $93 \mathrm{~K}$ met en évidence, quand on élève la température, deux transitions ayant des caractéristiques différentes :

- une cristallisation primaire relativement rapide et complète à $193 \mathrm{~K}$ qui s'accompagne d'une diminution de la transparence de l'échantillon. Cette transition correspond, compte tenu de nos conditions expérimentales, à la limite inférieure du domaine de germination homogène dont la limite supérieure se situe au voisinage de $338 \mathrm{~K}$ [11]. Elle se situe à une température bien inférieure à celle de la transition vitreuse $T_{\mathrm{G}}=248 \mathrm{~K}$ [14] mais au-dessus de la température de relaxation mécanique $T_{\mathrm{G}} \simeq 130 \mathrm{~K}$ associée à des oscillations localisées des groupements latéraux de la phase amorphe [15] : il est donc probable que ces types de mouvements, nécessaires pour que les groupements latéraux appartenant à des chaines voisines s'ordonnent, soient suffisants pour permettre la cristallisation du PB-1 sous la forme instable II. Ceci paraît d'autant plus vraisemblable ici que l'ordre local le long des chaînes est le même avant et après cristallisation, puisque les raies des spectres Raman de la phase amorphe et de la forme II apparaissent aux mêmes fréquences, ce qui est une règle générale observée lors de la cristallisation des polymères $[16,17]$

- une transformation forme II $\rightarrow$ forme I, lente et graduelle qui, toujours avec nos conditions expérimentales, débute vers $223 \mathrm{~K}$ et est pratiquement terminée après $48 \mathrm{~h}$ à $233 \mathrm{~K}$. Cette transition se situe à une température voisine de $T_{\mathrm{G}}$ [14]

La transformation II $\rightarrow$ I implique une diminution de volume de l'ordre de $10 \%$ ce qui est difficilement concevable si l'échantillon est vitreux; par contre la cristallisation sous forme II à partir du fondu n'implique qu'une variation de volume négligeable (Figs. 7 et 12, réf. [10]) et peut donc se faire au-dessous de $T_{\mathrm{G}}$.

Entre 193 et $223 \mathrm{~K}$ la phase II est la seule présente dans nos spectres; cela ne signifie pas cependant que cette phase est stable, à la pression ordinaire, entre ces deux températures mais plutôt que la cinétique de transformation II $\rightarrow$ I est si lente qu'elle n'est pas décelable par notre technique dans un temps limité à $48 \mathrm{~h}$. 


\section{Bibliographie}

[1] Natta, G., J. Polym. Sci. 16 (1955) 143.

[2] Natta, G., Corradini, P., Bassi, G. W., Makromol. Chem. 21 (1956) 240.

[3] Turner-Jones, A., J. Polym. Sci. B 1 (1963) 455.

[4] Holland, V. F., Miller, R. L., J. Appl. Phys. 35 (1964) 3241.

[5] Danusso, F., Gianotti, G., Makromol. Chem. 61 (1963) 139.

[6] Cornell, S. W., Koenig, J. L., J. Polym. Sci. A2 7 (1969) 1965.

[7] Boor, J., Mitchell, J. C., J. Polym. Sci. A 1 (1963) 59.

[8] Clampitt, B. H., Hugues, R. H., J. Polym. Sci. C 6 (1964) 43.

[9] Powers, J., Hoffman, J. D., Weeks, J. J., QuinN Jr, F. A., J. Res. Nat. Bur. Stand 69A (1965) 335.
[10] Vidotto, G., Kovacs, A. J., Kolloid Z. Z. Polym. 220 (1967) 1.

[11] Luongo, J. P., Salovey, R., J. Polym. Sci. A2 4 (1966) 997.

[12] Hendra, P. J., Jobic, H. P., Holland-Moritz, K., Polymer Letters Edit. 13 (1975) 365.

[13] Martin, L., Thèse de spécialité, Montpellier (1973)

[14] Polymer Handbook Interscience.

[15] Decroix, J. Y., Thèse, Lyon (1974).

[16] Birshtein, T. M., Ptitsyn., O. B., Conformation of Macromolecules (Interscience, New York) 1966.

[17] Rault, J., J. Macromol. Sci. Phys. B 15 (1978) 567. 